\title{
A year in the making
}

\author{
About 12 months into the COVID-19 pandemic, its immediate and lasting impacts on the climate system and fossil \\ fuel economy are now better understood. These insights will be fundamental to the global recovery - and ideally \\ the green transitions that accompany it - but the implementation will be hard-won.
}

T he date 11 March marks a year since COVID-19 was formally characterized as a pandemic by the World Health Organization (WHO). In addition to the colossal toll it has taken on human lives, the measures implemented to limit its spread have caused a seismic shift for many in how we live, work, travel and interact. These changes caused a decline in anthropogenic emissions and subsequent speculation of what this might mean for short- and long-term climate impacts. We think it is therefore a fitting time to reflect on what has been gleaned this past year on the climate impacts of COVID-19 safety measures and to highlight research, including several pieces in this month's issue, that gives insight into what is to come.

Last May, Corinne Le Quéré and co-authors reported daily $\mathrm{CO}_{2}$ emissions had decreased about $17 \%$ by early April 2020 compared to 2019 , as some of the first lockdowns were underway in major economies (Nat. Clim. Change 10, 647-653; 2020). In absolute emissions, these numbers were dominated by declines in the surface transport, power and industry sectors, though aviation exhibited the largest fractional emissions decrease. The researchers noted that the scale of these declines was likely temporary. Indeed, as economies began recovering, the gap narrowed to about $8.8 \%$ by mid-2020 (Nat. Commun. 11, 5172; 2020), and this slimmed further to about $7 \%$ as the year came to a close (Earth Syst. Sci. Data 12, 3269-3340; 2020).

What has become clear is that these impacts will not be enough to inch the physical climate system closer to the Paris Agreement goals. Work by Piers Forster and co-authors last August reported that by 2030, the impact of pandemic emissions decreases will result in a near-negligible cooling of about $0.01 \pm 0.005^{\circ} \mathrm{C}$ (Nat. Clim. Change 10, 913-919; 2020). These changes are a combination of decreases in emissions of $\mathrm{CO}_{2}$ and other climate forcers that can warm $\left(\mathrm{NO}_{x}\right)$ and cool $\left(\mathrm{SO}_{2}\right)$ the Earth system.

These longer-term impacts are imperative to consider as the 26th UN Climate Change Conference (COP26) approaches. Postponed for a full year due to COVID-19, this conference - scheduled for November

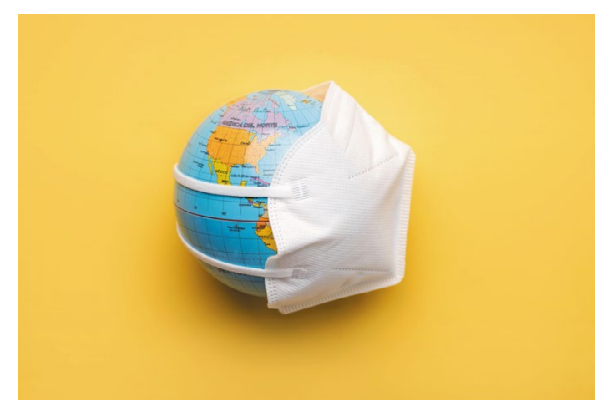

Credit: Esther Moreno / Alamy Stock Photo

2021 at the time of writing - will include the first five-year 'ratchet mechanism' as outlined in the Paris Agreement, in which national mitigation commitments are evaluated and tightened. In this issue, Le Quéré and colleagues build on their work from last May to place 2020 emissions in the context of the past five years, when Paris Agreement commitments began. The majority of high-income countries experienced emissions decreases during 2015-2019 (around 0.8\% per year), while upper-middle-income and lower-income countries primarily saw growth. By the end of 2020, however, COVID-19 impacts had caused all emissions to decrease - in middle-income countries by about $5 \%$, and in high- and lower-income countries by about $9 \%$. The authors point out that these impacts provide an opportunity to be strategic about longer-term changes to the energy sector.

Also in this issue, Christoph Bertram and co-authors evaluate changes to the global power sector from COVID-19, finding that decreased demand disproportionately affected coal power generation during 2020. The researchers estimate power sector $\mathrm{CO}_{2}$ emissions decreased by almost $7 \%$, and that after a 2020 dip, electricity demand is likely to stay at or below 2019 levels through the end of 2021. Combined with the likely continued expansion of low-carbon energy, this will increase the relative importance of renewables. These projections therefore suggest that a structural shift may be underway that signifies an inflection point in fossil fuel power generation. The authors contend that stricter policies on carbon pricing and fossil fuel subsidies are needed to buoy these impacts. But the upshot is hopeful: if policymakers galvanize these changes, COVID-19 impacts may have further shortened the lifespan of the fossil fuel economy.

So, what might the next five years look like? Yuli Shan and colleagues answer this question with an economic model of a post-pandemic world. The researchers create 27 scenarios that test a range of lockdown lengths and levels of strictness across 79 countries, estimating a global emissions reduction of $3.9-5.6 \%$ out to 2024 compared to a no-pandemic baseline scenario. While this implies some 'natural' emissions declines as a result of the pandemic itself, it is only enough to delay exceeding Paris Agreement targets. Importantly, the authors also consider a range of financial incentives and investment approaches that countries may use to counteract the pandemic's economic impacts. If these fiscal stimuli are carbon-intensive, a worst-case scenario estimates that emissions could increase as much as $16 \%$, reversing pandemic-related reductions. Alternatively, if investments in clean energy and other technologies are prioritized, emissions could decrease by an additional $4.7 \%$.

Taken together, these insights stress that the coming year is crucial in producing the time-sensitive climate action needed. Furthermore, while aspects of COVID19 can serve as a springboard for green transitions, these discussions will need to centre on fairness in carbon and emissions budgets. An obsession with renewable energy targets may sabotage their sustainability, for example (Nat. Clim. Change 10, 974-976; 2020), and expectations of carbon dioxide removal (Nat. Clim. Change 10, 640-646; 2020) or cumulative emissions allocations (Environ. Res. Lett. 14, 114039; 2019) on individual countries will require policies that don't place developing countries at a disadvantage. To ensure that these emissions decreases are sustained and just, expectations should be high for policymakers and COP26.

Published online: 3 March 2021

https://doi.org/10.1038/s41558-021-01010-Z 